This has a special reference to the problem stated above-that social progress demands the integration of science and society. When this statement is made to the ordinary person, he has difficulty in even seeing its meaning. But in a film we can show him, let us say, a bare spot on a bacteria culture, where a Penicillium spore hasfallen. We can show it to him through a microscope, and abstract ideas connected with it can be presented by a moving diagram; the scientific man's own deductions from this scene can be made 'real' by sound. The concept 'science' becomes something he can understand-in this case it may say "penicillin is bacteriostatic". The same film can also show wounded men being brought back from the coast of France and rejoining their families. The concept 'society' becomes also a real thing-ordinary people and their lives. The film can arrange its strips of celluloid so that the sounds and the pictures of these representations are linked together; science and society are shown to be integrated. The film brings to 'reality' something that is otherwise kut a mind picture, inaccessible to many. Its audiences gradually become 'citizen-scientists' in the sense that they grow to appreciate that science is part of their lives.

But the film medium, like all scientific tools, is powerful for ill as well as good. It does not necessarily tell the truth-indeed there are certain fundamental difficulties in the way of its doing so. The viewing and appraising of films which deal with science is therefore an important task. The lives of such scientific workers as Pasteur, Ehrlich, Faraday and Curie have been put upon the screen, and in very different ways. Most recently, psycho-analysis has received the attentions of Hollywood. Some of these films are good in their effect; others are bad. Since the public will get much of its ideas of scientific workers and of scientific method from the cinema, it is vital that those ideas should be soundly inspired. It is more than a matter of physics and chemistry.

${ }^{1}$ From "The Film in National Life", published 1932 by the Commission on Educational and Cultural Films. The Commission was originally brought into being through the agencies of the British Institute of Adult Education and the Association of Scientific Worke

${ }^{2}$ See Nature, 151, 718 (1943).

'Documentary News Letter, August 1942, article on "Scientific Films in Britain".

- Nature, 152, 745 (1943).

\section{THE MAXWELL LABORATORY AT THE UNIVERSITY OF MOSCOW}

\author{
BY PROF. V. ARKADIEV
}

$\mathrm{T}$ HE Maxwell Laboratory of Electromagnetism in the Physics Department of the Moscow State University was inaugurated twenty-five years ago, and this anniversary has been commemorated recently.

The work carried on in this Laboratory has dealt mainly with Maxwell's electromagnetic theory of light, with the view of the further development of its fundamental principles: (1) light as an electromagnetic phenomenon; (2) the optical properties of bodies (such as lustre, transparency, refraction of rays, etc.) can be computed in advance according to their electrical and magnetic properties. The laboratory work has established further the identity of light with electromagnetic waves and the analogy existing between the two latter aspects of waves. In 1922 the Laboratory discovered a new source of radiation, the mass-radiator; this enables one to obtain intermediate ultra-Herzian waves, which form a connecting link between radio- and heat-waves. In 1934 special plates, sensitive to Herzian waves, were invented and prepared in the Laboratory; these plates have made possible the application in radio engineering of methods employed in photography (stictography), namely, those of fixing the traces left by radio waves upon paper.

Shortly before the War, the Laboratory demonstrated the possibility of using radio waves for radioscopy and suggested special screens, luminescent under the action of centimetre waves, similar to those used for X-rays. In the course of the further development of Maxwell's theory, the Laboratory has elaborated a comprehensive theory of 'passive' spectra, the most interesting result of this theory being the application of spectral analysis to the study of the magnetism of bodies; this involves the application of methods of mathematical analysis of optical absorption spectra to the investigation of the process of magnetization, particularly of the magnetization of technical magnetic materials.

The theory of passive spectra has been applied to the behaviour of matter of every description, beginning with the ionosphere and gases and ending with its coarser aspects, such as resin, cast iron, ores and rocks.

The combination of Maxwell's electromagnetic equations with the laws of motion established by Newton affords the possibility of obtaining general equations, representing a scheme of the behaviour of matter along the entire scale of electromagnetic waves. Among other things the scheme enables us to deduce the dispersion of Debye's dielectrics, the mag. netic dispersion of paramagnetics and Compton's formula for the refraction coefficient for X-rays. An analogous inference is obtained for the magnetic pro. perties of ferromagnetic bodies in the region of the ultra-Herzian waves, where the magnetic spectrum of ferromagnetic bodies is transferred into its own 'Röntgen' region in which, owing to the high frequency of the vibrations, only insignificant vestiges of the magnetic properties can remain.

\section{AXIS ORIENTATION OF QUARTZ CRYSTALS}

A $\mathrm{N}$ article by G. W. Willard (Bell Lab. Rec., 22, A No. 7; March 1944) deals with the methods used in inspecting quartz crystals and in deter mining the axis orientation. In the original crystallization of quartz, foreign substances, such as other minerals or bubbles of gas or liquid, may be included, and part of the inspection procedure is undertaken to locate such inclusions so that they may be cut away. One of the dangers of using plates with inclusions is that the resulting discontinuities in their elastic and thermal properties may cause them to crack under the influence of temperature changes. Another common defect is the presence of cracks, due either to the effect of inclusions or to the rough treatment the quartz receives in river beds or in being broken from its natural formation. These cracks may be completely internal and very fine, and thus not 